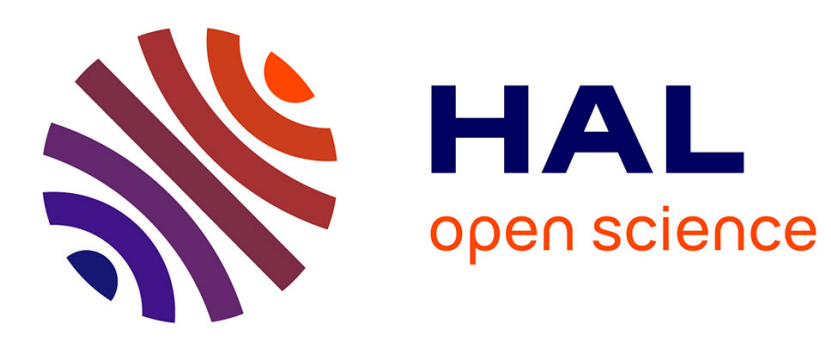

\title{
Multimodal coupling of periodic lattices and application to rod vibration damping with a piezoelectric network
}

Boris Lossouarn, Mathieu Aucejo, Jean-François Deü

\section{To cite this version:}

Boris Lossouarn, Mathieu Aucejo, Jean-François Deü. Multimodal coupling of periodic lattices and application to rod vibration damping with a piezoelectric network. Smart Materials and Structures, 2015, 24 (4), 10.1088/0964-1726/24/4/045018 . hal-01691077

\section{HAL Id: hal-01691077 \\ https://hal.science/hal-01691077}

Submitted on 22 Mar 2018

HAL is a multi-disciplinary open access archive for the deposit and dissemination of scientific research documents, whether they are published or not. The documents may come from teaching and research institutions in France or abroad, or from public or private research centers.
L'archive ouverte pluridisciplinaire HAL, est destinée au dépôt et à la diffusion de documents scientifiques de niveau recherche, publiés ou non, émanant des établissements d'enseignement et de recherche français ou étrangers, des laboratoires publics ou privés. 


\title{
Multimodal coupling of periodic lattices and application to rod vibration damping with a piezoelectric network
}

\author{
B Lossouarn ${ }^{1}$, M Aucejo ${ }^{1}$, J-F Deü ${ }^{1}$ \\ 1 Structural Mechanics and Coupled Systems Laboratory, Conservatoire \\ National des Arts et Métiers, 2 Rue Conté, 75003, Paris, France \\ E-mail: boris.lossouarn@cnam.fr
}

\begin{abstract}
Elastic lattice of point masses can be a suitable representation of a continuous rod for the study of longitudinal wave propagation. By extrapolating the classical tuned mass damping strategy, a multimodal tuned mass damper is introduced from the coupling of two lattices having the same modal properties. The aim of the study is then to implement this multimodal control on a rod coupled to an electrical network. The electromechanical analogy applied to a lattice gives the required network and the energy conversion is performed with piezoelectric patches. The coupled problem is modeled by a novel semicontinuous transfer matrix formulation, which is experimentally validated by a setup involving a rod equipped with 20 pairs of piezoelectric patches. The broadband efficiency of the multimodal control is also experimentally proved with vibration reductions up to $25 \mathrm{~dB}$ on the four first resonances of the rod. At last, the practical interest of the network is pointed out as it limits the required inductance. This is confirmed by the present purely passive setup that only involves standard low value inductors.
\end{abstract}

PACS numbers: 85.50.-n, 63.10.+a, 46.40.Ff, 84.32.-y, 46.70.Hg

Keywords: vibration control, multimodal coupling, lattices, passive damping, piezoelectricity, shunt, electrical network

Submitted to: Smart Mater. Struct. 


\section{Introduction}

Wave propagation in one dimensional periodic structures can be analyzed with the transfer matrix formulation. As described by Mead [1], this approach is based on the analysis of a unit cell, which periodically repeats with the same mechanical properties. Both mechanical states at the ends of the unit cell respect a relation represented by a constant transfer matrix. This relation is then propagated in order to obtain the global behavior of a finite structure. This applies to a rod but also to its discrete model which can be represented by a lattice of point masses. The periodic lattices were presented by Brillouin [2], who gave several fundamental results related to wave propagation in mechanical as well as in electrical periodic structures. An electromechanical analogy between lattices of different nature was thus already introduced. This analogy was formalized by Bloch [3] and Beranek [4] for more general structures. All of this gives useful elements to find the analogues of coupled problems involving electrical components fitted on several piezoelectric patches [5]. First, it is seen that a resonant shunts can be apprehended as a tuned mass damper $[6,7]$. By tuning the electrical components, vibration reduction of a structure is expected [8]. This concept was applied by Thorp et al. for the damping of longitudinal waves in a rod periodically covered with piezoelectric patches [9]. The use of the transfer matrix formulation allows studying the propagation constants as well as the global behavior of the coupled structure. It was then extended to the study of transverse waves by taking into account the influence of various shunts either passive as the resonant ones $[10,11,12,13]$ or active as those presenting negative capacitors [14]. However, just a limited number of papers consider electrical connections otherwise than independently between the different piezoelectric patches. One can yet mention the work of Yu et al. [15] who analyzed the use of an electric network in order to delocalize vibration in a periodic blade assembly. For the damping of vibration, dell'Isola et al. introduced several principles on the influence of distributed piezoelectric networks on transverse wave propagation $[16,17,18]$. From a continuous formulation, one optimal architecture appears as the electric analogue of a beam [19]. Nevertheless, none of the aforementioned studies on electrical networks benefits from the transfer matrix method. This was still performed by $\mathrm{Lu}$ and Tang who added electrical components in state vectors for the analysis of a periodic network [20].

The present work deals with the above mentioned concepts in order to present a passive and multimodal damping strategy that can apply to vibration damping of a rod. First of all, the transfer matrix formulation is presented and applied to a continuous rod which is then discretized into a lattice of point masses. The differences between the continuous model and the lattice model are then clarified. Considering a finite lattice, two control strategies based on modal coupling are presented for the purpose of converting the vibration energy of a main structure into a damping device. The first resonance-based solution involves independent tuned mass dampers, while the second solution considers the coupling of the main lattice to a damping lattice having the same modal properties. An original multimodal tuned mass damper is thus presented with this second strategy. Moreover, both control strategy can be expressed for electrical networks by using the direct electromechanical analogy $[3,4]$. From this analogy, it is introduced a parallel between the two discrete control strategies and coupled problems involving piezoelectric patches. For example, independent tuned mass dampers distributed on a lattice are the analogues of distributed resonant shunts on a rod [9]. Consequently, the next step is to implement the multimodal stategy by connecting the rod to an electrical network having similar modal properties. This network comes from the electrical analogue of a lattice, which is represented by a line of inductors with connection to the ground through capacitors. The coupling to a rod through piezoelectric patches is then modeled by focusing on global properties of a single unit cell. This transfer matrix formulation considerably simplify the analysis compared to former analytic formulations where the electrical network was seen as a continuum $[18,21]$. Here, it is presented a global semi-continuous transfer matrix that keep the natural discretization of the electrical network together with the continuity of the rod. This model is experimentally validated by analyzing the effect of the two control strategies on electrical and mechanical frequency response functions for the four first modes of the rod. Moreover, another main result concerns the experimental validation of the multimodal damping strategy. With a simple modal coupling condition obtained from the discrete models, the efficiency of the method is clearly proved with reductions up to $25 \mathrm{~dB}$ on the resonances of 
the considered frequency range. Another interest of this control strategy is the possibility to limit the value of the required inductors. This offers a chance to implement a completely passive control, as it was performed in the present experimental setup.

\section{Wave propagation in a discretized rod}

The transfer matrix formulation is introduced in order to analyze vibrations of one-dimensional structures. The classical rod model is considered before looking at its lattice equivalent. The differences between the two models are then clarified.

\subsection{Transfert matrix formulation}

As presented by Mead, a one-dimensional periodic structure can be seen as a succession of identical unit cells [1]. Each unit cell presents a relation between the mechanical states at its two ends, which is described by a transfer matrix $\boldsymbol{T}$ :

$$
\left[\begin{array}{c}
\boldsymbol{q}_{\mathrm{R}} \\
\boldsymbol{F}_{\mathrm{R}}
\end{array}\right]=\boldsymbol{T}\left[\begin{array}{c}
\boldsymbol{q}_{\mathrm{L}} \\
\boldsymbol{F}_{\mathrm{L}}
\end{array}\right] \text {. }
$$

The components of the state vectors, $\boldsymbol{q}$ and $\boldsymbol{F}$, refer to the displacements and forces at positions defined by the subscripts $\mathrm{L}$ or $\mathrm{R}$ for the left or right ends of the unit cell. $\boldsymbol{F}_{\mathrm{R}}$ is the force applied on the considered cell by its right neighbor and $\boldsymbol{F}_{\mathrm{L}}$ is the force applied by the considered unit cell on its left neighbor. This convention allows keeping the continuity of the state vector which is obvious for the displacements but not for the forces.

By definition, each unit cell of a periodic structure presents the same transfer matrix $\boldsymbol{T}$. Then, from the continuity of the state vector, the mechanical state at a position 0 is propagated to the right of the $n^{\text {th }}$ unit cell by raising $\boldsymbol{T}$ to the power of $n$ :

$$
\left[\begin{array}{c}
q_{n} \\
F_{n}
\end{array}\right]=T^{n}\left[\begin{array}{c}
q_{\mathrm{o}} \\
F_{\mathrm{o}}
\end{array}\right]=\left[\begin{array}{cc}
T_{q q}^{n} & T_{q F}^{n} \\
T_{F q}^{n} & T_{F F}^{n}
\end{array}\right]\left[\begin{array}{c}
q_{\mathrm{o}} \\
F_{\mathrm{o}}
\end{array}\right]
$$

For a finite structure, the solution of the problem requires to consider the boundary conditions. For example, with a prescribed force $\boldsymbol{F}_{\mathbf{o}}$ applied to the left end of a free-free structure, the displacement $\boldsymbol{q}_{\boldsymbol{n}}$ at its right end is defined by:

$q_{n}=\left(T_{q F}^{n}-T_{q q}^{n} T_{F q}^{n-1} T_{F F}^{n}\right) F_{\mathrm{o}}$

\subsection{Mechanical analysis of a rod}

For a one-dimensional medium of Young modulus $Y$ and density $\rho$, the longitudinal wave equation can be expressed in the following form:

$\rho \frac{\partial^{2} u(x, t)}{\partial t^{2}}=Y \frac{\partial^{2} u(x, t)}{\partial x^{2}}$,

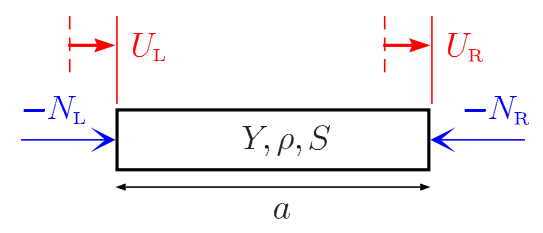

Figure 1. Considered rod portion.

where $u(x, t)$ refers to the longitudinal displacement. A space-time separation $u(x, t)=U(x) g(t)$ gives the harmonic solutions of the wave equation (4) and the dispersion relation:

$\omega^{2}=\frac{Y}{\rho} k^{2}$,

where $\omega$ is the angular frequency and $k$ is the wavenumber. The longitudinal displacement amplitude of the rod portion presented in figure 1 is given by:

$U(x)=U_{\mathrm{L}} \cos (k x)+\frac{U_{\mathrm{R}}-U_{\mathrm{L}} \cos (k a)}{\sin (k a)} \sin (k x)$,

where $a$ is the length of a rod portion and the subscripts $L$ and $R$ refer respectively to the positions $x=0$ and $x=a$.

The normal force into the rod is proportional to the first derivative of the displacement presented in (6), i.e. $N(x)=Y S U^{\prime}(x)$, where $S$ is the cross-section area. Consequently, the end forces are linearly obtained from the end displacements of the rod portion. The two equations can be rearranged into the following matrix form:

$$
\left[\begin{array}{c}
U_{\mathrm{R}} \\
N_{\mathrm{R}}
\end{array}\right]=\left[\begin{array}{cc}
\cos (k a) & \frac{1}{Y S k} \sin (k a) \\
-Y S k \sin (k a) & \cos (k a)
\end{array}\right]\left[\begin{array}{c}
U_{\mathrm{L}} \\
N_{\mathrm{L}}
\end{array}\right],
$$

This corresponds to the transfer matrix formulation described in (1), the state vectors containing the longitudinal displacement $U$ and the normal force $N$. At the end, considering a free-free rod of length $l=n a$ excited at one end, it is possible to get the frequency response of the displacement at the other end from (3).

\subsection{Lattice model}

The purpose of the previous formulation is not obvious for an homogeneous rod as the global solution can be obtained directly from the analysis of a single unit cell of length $l$ (with $n=1$ ). Nevertheless, the transfer matrix formulation becomes useful when looking at periodic models where the global layout is not identical to the local one. For the propagation of longitudinal waves, Brillouin analyzed the periodic lattice model as a discrete representation of a continuous medium [2]. This applies to a rod by considering periodically lumped masses $m$ linked serially by springs of identical stiffness $K$. The unit cell can thus be represented by two half masses at each ends of a spring as shown in figure 2. The mass and the stiffness are determined 


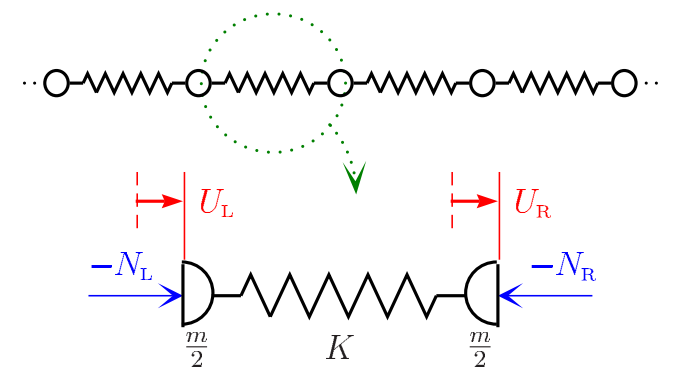

Figure 2. Lattice of point masses and a corresponding unit cell.

from the local properties of the rod and the length $a$ of the unit cell:

$m=\rho S a \quad$ and $\quad K=\frac{Y S}{a}$.

The mechanical analysis of the discrete unit cell gives two equations involving the longitudinal displacement and the normal force at both ends. This can be presented with the transfer matrix formulation:

$$
\left[\begin{array}{c}
U_{\mathrm{R}} \\
N_{\mathrm{R}}
\end{array}\right]=\left[\begin{array}{cc}
1-f & \frac{1}{K} \\
-2 K f\left(1-\frac{f}{2}\right) & 1-f
\end{array}\right]\left[\begin{array}{c}
U_{\mathrm{L}} \\
N_{\mathrm{L}}
\end{array}\right],
$$

where $f=\omega^{2} m /(2 K)$. Since the eigenvalues $\lambda$ of this transfer matrix are related to the equivalent wavenumber $\bar{k}$ of the discrete problem by $\lambda=$ $\exp ( \pm \mathrm{j} \bar{k} a)$, one gets the following non-linear dispersion relation when $\bar{k} a \leq \pi$ :

$\omega^{2}=\frac{Y}{\rho}\left(\frac{\sin \left(\frac{\bar{k} a}{2}\right)}{\frac{\bar{k} a}{2}}\right)^{2} \bar{k}^{2}$.

Already introduced in [2], this equation is here obtained from the transfer matrix and it can be compared to the rod dispersion relation presented in (5). The two relations are equivalent when the product $\bar{k} a$ goes to zero, i.e. when the size of the unit cell is sufficiently small compared to the considered wavelength. As a reference, it can be seen from (10) that with 10 unit cells per wavelength $(\bar{k} a=$ $2 \pi / 10)$, the frequency obtained with the discrete model differs by less than $2 \%$ from the one got with the rod dispersion relation. In any case, by comparing (7) and (9), it is seen that it is still possible to convert the lattice into its initial continuous model by applying the following reverse transformation:

$f \rightarrow 1-\cos (k a)$ and $K \rightarrow \frac{Y S}{a} \frac{k a}{\sin (k a)}$.

Consequently, all the previous elements give a way to study the mechanical behavior of a rod as well as its discrete equivalent. The difference between those two models can be evaluated and the conversion from one to the other is clarified in both ways.

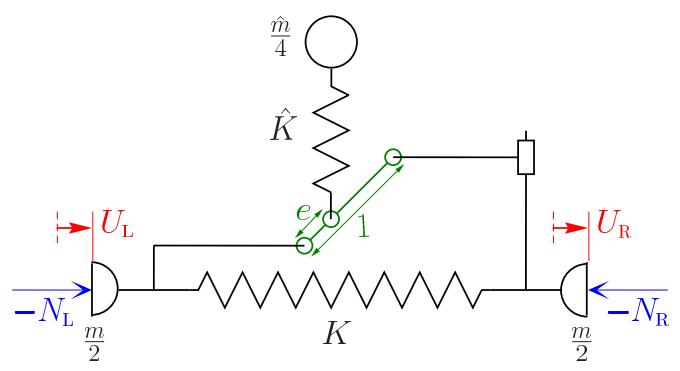

Figure 3. A tuned mass damper connected to each unit cell.

\section{Vibration control based on modal coupling}

When considering vibration of a structure, passive control have the benefit of being self-sufficient and unconditionally stable. It is presented two resonancebased passive strategies for the purpose of attenuate vibration of a main lattice. The first strategy involves several identical tuned mass dampers while the second presents a multimodal coupling. Both solutions have electrical analogues, which present similar transfer matrices.

\subsection{Independent tuned mass dampers}

A way to limit vibration of a structure is to add multiple control devices locally. When looking at passive solutions, the tuned mass damping strategy is suitable to convert vibration energy from a main structure to the added masses [6]. This energy transfer applies around a specific frequency, which is chosen by tuning the resonance of the added systems. The strategy can be implemented in a periodic lattice by distributing identical tuned mass systems as it was introduced in the Vincent's model [2]. A variant is presented in figure 3 , where a strain in the main structure is converted into a displacement of the added system thanks to a lever of $e$ ratio. This stresses the spring of stiffness $\hat{K}$ which put the tuned mass $\hat{m} / 4$ in motion. In all the following study, the hatted symbols characterize the control device and the nonhatted symbols refer to the main structure that need to be controlled.

As achieved previously for the basic unit cell, the mechanical analysis of this new sub-structure can be realized for the purpose of getting the transfer matrix formulation of the problem. The following system of equations is obtained for small displacements:

$$
\left[\begin{array}{c}
U_{\mathrm{R}} \\
N_{\mathrm{R}}
\end{array}\right]=\left[\begin{array}{cc}
1-f \tilde{f} & \frac{\tilde{f}}{K} \\
-2 K f\left(1-\frac{f \tilde{f}}{2}\right) & 1-f \tilde{f}
\end{array}\right]\left[\begin{array}{c}
U_{\mathrm{L}} \\
N_{\mathrm{L}}
\end{array}\right]
$$

where $\tilde{f}=\frac{1-\omega^{2} \frac{\hat{m}}{4 \bar{K}}}{1-\omega^{2} \frac{\hat{m}}{4}\left(\frac{e^{2}}{K}+\frac{1}{\tilde{K}}\right)}$.

When $e=0$, it can be seen that $\tilde{f}=1$ and the transfer matrix becomes equal to the one presented in 
(9). Without coupling, the added system has no effect and the structure behaves as a simple lattice. Then, for $e \neq 0$ and $\omega=2 \sqrt{\hat{K} / \hat{m}}$, which correspond to the natural frequency of the added spring-mass systems, $\tilde{f}$ becomes equal to zero. Therefore, $U_{\mathrm{R}}=U_{\mathrm{L}}$ for every unit cells. The displacement is the same in all the primary lattice and no strain is observable whatever the boundary conditions.

The independent tuned mass damping strategy can be applied to a $l=1 \mathrm{~m}$ free-free duralumin rod. Its Young modulus is $Y=73.9 \mathrm{GPa}$, its density $\rho=2780$ $\mathrm{kg} / \mathrm{m}^{3}$ and the cross-section area is set to $S=4 \mathrm{~cm}^{2}$. This finite structure is discretized in a lattice of $n=20$ unit cells and each one is fitted with an identical tuned mass damper, as presented in figure 3 . The added mass $\hat{m} / 4$ is then set to one tenth of the mass $m$ and $\hat{K}$ is tuned so that the natural frequency of the spring-mass systems corresponds to the first natural frequency of the main structure.

The right end velocity is computed from (3) and (12) by applying an harmonic unit force on the left end of the lattice. The results are shown in figure 4, where three cases are presented. The first one corresponds to a situation with a lever ratio $e$ equal to 0 . The lattice is vibrating without any effect of the added system and the four first natural frequencies are displayed. As no damping is added in the model, the velocity goes naturally to infinity at those particular frequencies. The second case is obtained with $e=1$ and again without damping. Around the first natural frequency the initial resonance is no more observable as the energy is transferred to the added masses. Yet, new resonances appear on either side of the previous one, as it is classically observed when using undamped tuned mass systems [6]. The amplitude of those new resonances can be reduced by introducing damping. The solution provided in most of the tuned mass strategies consists in adding a damper in parallel to the springs of stiffness $\hat{K}$. However, it is also possible to consider a damper linking directly the tuned masses to the ground [7]. It is this last solution which is implemented in the third case by replacing the tuned masses $\hat{m} / 4$ by $\hat{m}^{\star} / 4=\hat{m} / 4-j \hat{c} / \omega$, where $\hat{c}$ is the viscous coefficient of the added dampers. With $\hat{c}=5 \mathrm{~N} /(\mathrm{m} / \mathrm{s})$, the velocity amplitude around the first resonance is reduced to $-50 \mathrm{~dB}$. This illustrates the efficiency of the independent tuned mass dampers for a control focusing on one particular resonance.

\subsection{Multimodal tuned mass damper}

The previous control strategy consists in connecting identical unimodal devices to a multimodal structure. By extension, it is possible to consider a coupling between two multimodal structures. This concept can

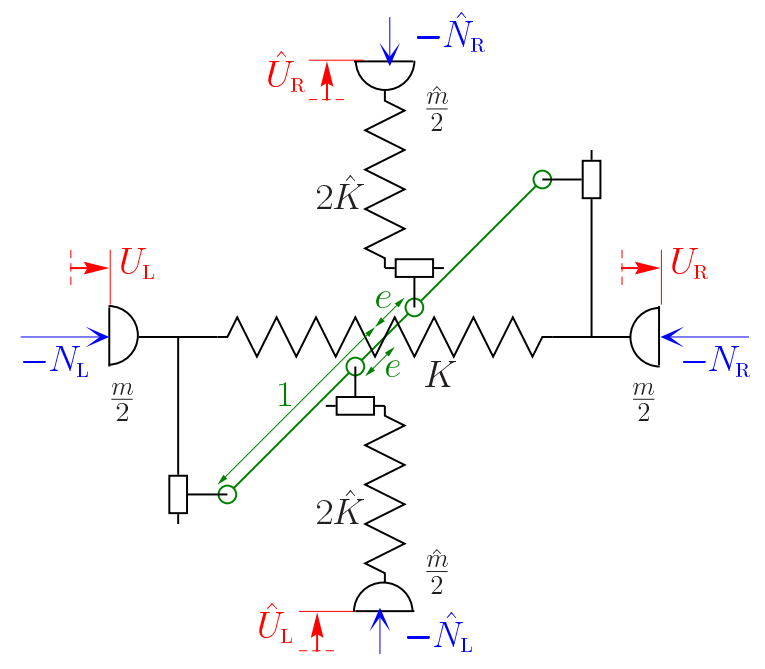

Figure 5. Unit cell for the coupling of two lattices.

be illustrated by analyzing two lattices involving the same number of unit cells. The main lattice is the structure that need to be controlled and the other one is considered as a multimodal control device. If all the natural frequencies of the control device are simultaneously tuned to those of the main structure, this added system should behave as a multimodal tuned mass damper.

The first step is to find a way to mechanically connect the two lattices in order to allow energy transfers between facing pairs of unit cells. A solution is presented in figure 5 as a generalization of the tuned mass damper architecture of figure 3 .

The fundamental difference is that the added masses are no more free but are inherent components of the secondary lattice. When the lever ratio $e$ is equal to 0 , the two lattices of $n$ unit cells are completely independent. On the contrary, if $e$ is different to zero, they interact through the $n$ lever mobilities. The study of the global problem requires firstly to focus on the unit cell presented in figure 5. A mechanical analysis for small displacements leads to the following formulation:

$\left[\begin{array}{c}U_{\mathrm{R}} \\ \hat{U}_{\mathrm{R}} \\ N_{\mathrm{R}} \\ \hat{N}_{\mathrm{R}}\end{array}\right]=\left[\begin{array}{cccc}1-f & e \frac{\hat{K} \hat{K}}{K} & \frac{1}{K} & -\frac{e}{K} \\ e f & 1-\Lambda \hat{f} & -\frac{K}{K} & \frac{1}{K} \\ -2 K f\left(1-\frac{f}{2}\right) & -e \hat{K} f \hat{f} & 1-f f & e f \\ -e \hat{K} f \hat{f} & -2 \hat{K} \hat{f}\left(1-\frac{\Lambda \hat{f}}{2}\right) & e \frac{\hat{K}}{K} \hat{f} & 1-\Lambda \hat{f}\end{array}\right]\left[\begin{array}{c}U_{\mathrm{L}} \\ \hat{U}_{\mathrm{L}} \\ N_{\mathrm{L}} \\ \hat{N}_{\mathrm{L}}\end{array}\right]$,

where $f=\omega^{2} m /(2 K), \hat{f}=\omega^{2} \hat{m} /(2 \hat{K})$ and $\Lambda=$ $1+e^{2} \hat{K} / K$. By forcing $\hat{N}_{\mathrm{L}}$ and $\hat{N}_{\mathrm{R}}$ to zero, it is verified that the unit cell of figure 3 is a particular case of the one illustrated in figure 5. Indeed, the $4 \times 4$ transfer matrix can then be condensed in a $2 \times 2$ matrix which is equal to the matrix presented in (12).

After having connected the two lattices, a second step consists in tuning the added lattice to suit the modal properties of the main one. It can be seen 


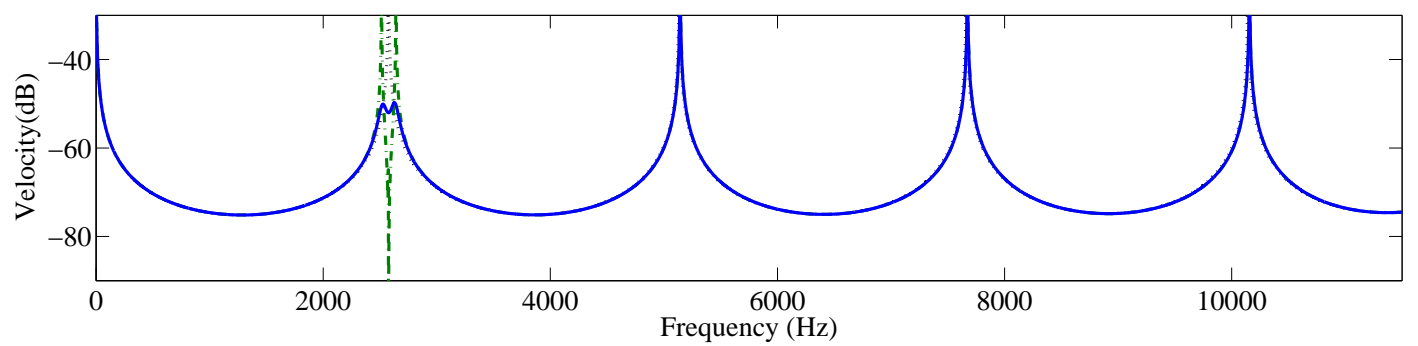

Figure 4. Frequency response functions with independent tuned masses $-(\cdots)$ for $e=0,(-\cdot-)$ for $e=1$ and $\hat{c}=0,(-)$ for $e=1$ and $\hat{c}=5$.

from (9) that a same mass-stiffness ratio and identical boundary conditions lead to a similar eigenmode problem for the two lattices. In this situation, all the natural frequencies and mode shapes are identical in the two structures. In order to show the effect of the modal coupling, the example of a free-free lattice with $n=20$ unit cells is again considered. The mass $\hat{m}$ is set to $m / 100$ and the modal coupling condition on the mass-stiffness ratio gives:

$\hat{K}=\hat{m} \frac{K}{m}$.

Considering the boundary conditions, the end forces of the secondary lattice, $\hat{N}_{0}$ and $\hat{N}_{n}$, are set to zero in order to satisfy the equivalence with the main free-free structure.

The $4 \times 4$ transfer matrix is used to compute the frequency response function of the right end velocity in the main lattice. The results of the multimodal coupling are shown in figure 6 . When $e$ is equal to zero, the frequency response function is the same as the one obtained with the independent tuned mass dampers. Indeed, no energy is transferred to the added masses which have no effect on the main lattice. However, the shape of the curve is clearly different when $e$ differs from 0 . A tuned mass effect is then observable on either side of all the initial natural frequencies. As mentioned previously, the amplitudes can be reduced by adding damping in the control system. Again, replacing $\hat{m}$ by $\hat{m}^{\star}=\hat{m}-j \hat{c} / \omega$ is equivalent to the addition of dampers between the ground and the masses of the secondary lattice. With $\hat{c}=1$, it is seen that the velocity is strongly limited whatever the considered frequency. This highlights the interest of the multimodal tuned mass damping strategy compared to the unimodal strategy. It is still remarked that the modal coupling condition presented (12) doesn't take into account the influence of the damping parameter. This induces observable errors compared to an optimal configuration as the damping slightly shifts the resonances of the secondary lattice. Nevertheless, as seen in the following, this shift remains limited compared to the one introduced by the reverse transformation to a primary continuous structures.

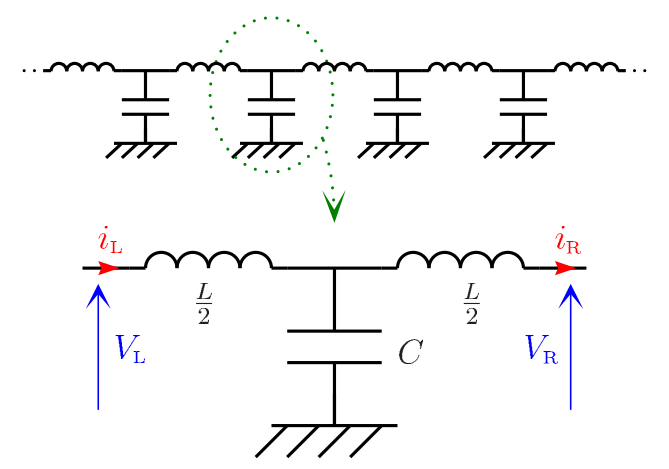

Figure 7. Electrical analogue of a lattice and corresponding unit cell.

\subsection{Electrical analogues}

All the previous lattice models can be transposed to electrical architectures by applying the direct electromechanical analogy $[3,4]$. This analogy assumes the equivalence between force and voltage and between velocity and intensity. Consequently, a mass is equivalent to an inductance and a stiffness to the inverse of a capacitance. Thereby, a lattice of point masses corresponds to an electrical network made of a line of inductors with connections to the ground through capacitors [2]. This is illustrated in figure 7 together with the corresponding electrical unit cell.

When comparing the mechanical and the electrical unit cells, it can be seen that the analogues of the left and right intensities are directly the derivatives of the left and right displacements. Yet, the two voltages are equivalent to the opposite of the normal forces in order to respect their definition. The resulting equations describing the electrical behavior are then identical to the mechanical ones by replacing $m$ by $L$ and $K$ by $1 / C$. Consequently, from (9), the following transfer matrix formulation is obtained:

$\left[\begin{array}{c}i_{\mathrm{R}} \\ V_{\mathrm{R}}\end{array}\right]=\left[\begin{array}{cc}1-f & -j C \omega \\ \frac{2}{\mathrm{j} C \omega} f\left(1-\frac{f}{2}\right) & 1-f\end{array}\right]\left[\begin{array}{c}i_{\mathrm{L}} \\ V_{\mathrm{L}}\end{array}\right]$,

where $f=\omega^{2} L C / 2$.

As for the mechanical lattices, it is possible to consider the coupling of two electrical networks. In 


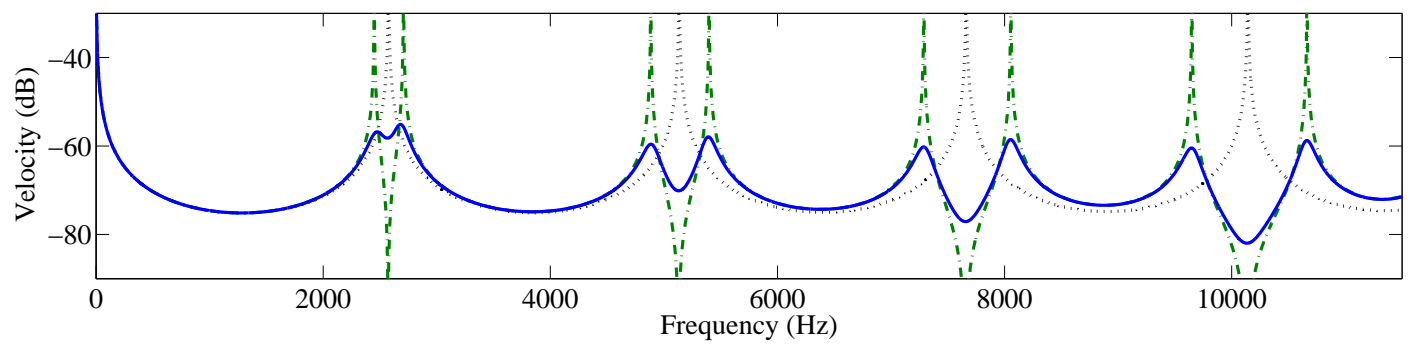

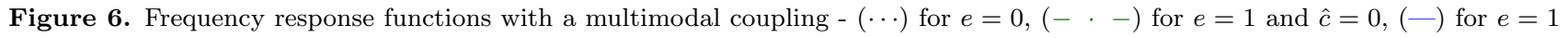
and $\hat{c}=1$.
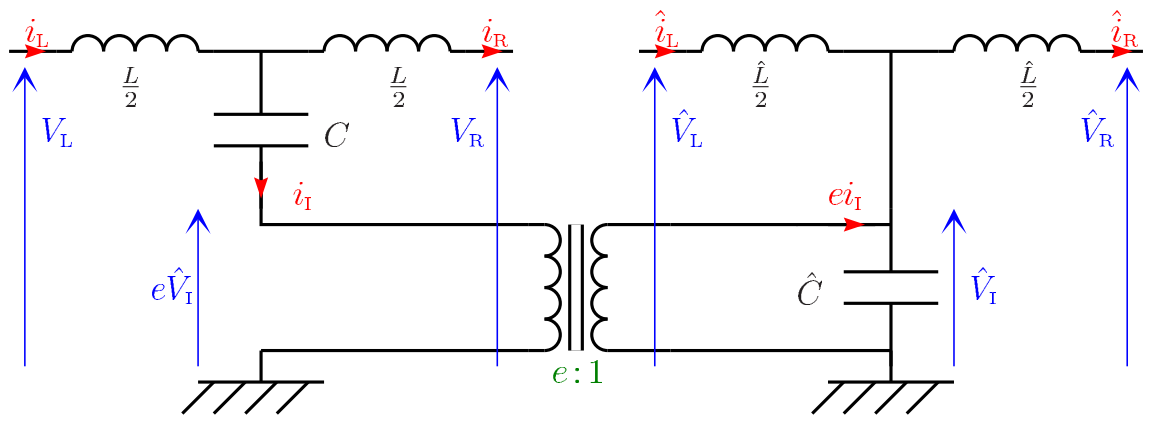

Figure 8. Unit cell for the coupling of two electrical networks.

this way, the analogue of the coupled mechanical unit cell of figure 5 is presented in figure 8. A transformer of $e$ ratio transfers the energy from a network to the other. This is indeed the analogue of the lever represented in the mechanical model. Consequently, with the analogies $L \leftrightarrow m, C \leftrightarrow 1 / K, i \leftrightarrow j \omega U$ and $V \leftrightarrow-N$, the electrical equations are the same as when coupling two mechanical lattices. The electric transfer matrix corresponding to the coupling of two networks can thus be easily deduced from (13). Then, by setting $\hat{V}_{\mathrm{L}}=\hat{V}_{\mathrm{R}}=0$ in the electric scheme of figure 8, it appears the electrical analogue of a tuned mass damper. Here, it consists of a capacitor $\hat{C}$ and an inductor $\hat{L} / 4$ in parallel. Finally, this last configuration is equivalent to the mechanical unit cell fitted with a tuned mass damper as it was illustrated in figure 3. All the presented discrete mechanical models can thus be transposed into their analogue electrical problems. The corresponding transfer matrices are described and can be used to study wave propagation in an electrical network. The unimodal and multimodal control strategies still applies and could give the possibility to avoid electric line resonances.

\section{Application to piezoelectric damping}

The two resonance-based control solutions are extended to the damping of a rod covered with distributed piezoelectric patches. The damping systems are build with electrical components and the piezoelec- tric elements take the role of connecting the rod to the electrical structure at the level of each unit cell. We obtain an electromechanical problem, which can be described with a transfer matrix formulation.

\subsection{Global piezoelectric model}

A connection of a mechanical structure to an electrical structure requires a component capable of converting mechanical energy into electric energy and reciprocally. Piezoelectric material fulfills this function as it links a strain to an electric field through its direct and reverse effects. It is then considered a transversely polarized piezoelectric rod under plane stress assumption. With ' 1 ' referring to the longitudinal direction and ' 3 ' to the direction of polarization, the 3D linear formulation can be simplified into the following one-dimensional stresscharge form [22]:

$\left\{\begin{array}{l}\sigma_{1}=\bar{c}_{11}^{E} \varepsilon_{1}-\bar{e}_{31} E_{3} \\ D_{3}=\bar{e}_{31} \varepsilon_{1}+\bar{\epsilon}_{33}^{\varepsilon} E_{3}\end{array}\right.$,

where $\bar{c}_{11}^{E}=1 / s_{11}^{E}, \quad \bar{e}_{31}=d_{31} / s_{11}^{E}$ and $\bar{\epsilon}_{33}^{\varepsilon}=$ $\epsilon_{33}^{\sigma}-d_{31}^{2} / s_{11}^{E} . \quad \sigma_{1}$ and $\varepsilon_{1}$ represents the longitudinal stress and strain, $D_{3}$ and $E_{3}$ are the transverse electric displacement and electric field. Concerning the material constants, $s_{11}^{E}$ is the elastic compliance at constant electric field, $d_{31}$ is the piezoelectric charge constant and $\epsilon_{33}^{\sigma}$ is the permittivity at constant stress. Those constants are here highlighted because they are commonly used by the piezoelectric material suppliers. 


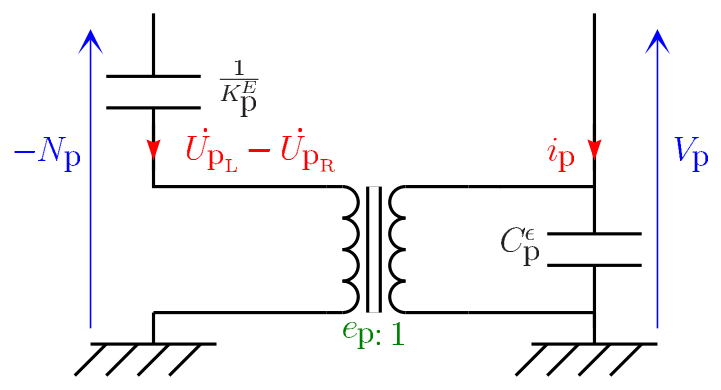

Figure 9. Global model of the piezoelectric element.

Regarding a thin piezoelectric patch vibrating along its longitudinal direction, if its length $l_{\mathrm{p}}$ is sufficiently small compared to the considered wavelength, the strain $\varepsilon_{1}$ can be seen as uniform over $l_{\mathrm{p}}$. Consequently, the strain is defined thanks to a difference between the positions of the left and right ends of the element: $\varepsilon_{1}=\left(U_{\mathrm{p}_{\mathrm{R}}}-U_{\mathrm{p}_{\mathrm{L}}}\right) / l_{\mathrm{p}}$. The electric field $E_{3}$ can also be regarded as a constant for a small thickness $h_{\mathrm{p}}[22] . \quad E_{3}$ is then expressed from $\hat{V}_{\mathrm{p}}$, the voltage between the two electrodes, by $E_{3}=-\hat{V}_{\mathrm{p}} / h_{\mathrm{p}}$. Then, if $b$ is the width of the patch, $S_{\mathrm{p}}=h_{\mathrm{p}} b$ is its cross-section area. Considered uniform in the cross-section, the longitudinal stress is thus obtained by $\sigma_{1}=N_{\mathrm{p}} / S_{\mathrm{p}}$, $N_{\mathrm{p}}$ being the normal force applied to the patch. At last, the intensity $\hat{i}_{\mathrm{p}}$ passing through the electrodes of surface area $A_{\mathrm{p}}=l_{\mathrm{p}} b$ is related to the derivative of the charge displacement $D_{3}$. This is expressed by $D_{3}=-\hat{i}_{\mathrm{p}} / j A_{\mathrm{p}} \omega$. The sign of the intensity is defined relatively to the voltage $\hat{V}_{\mathrm{p}}$ by considering a passive sign convention for the piezoelectric element. Consequently, the local piezoelectric formulation (16) gives a system of equations where appears the global variables of the considered patch:

$\left\{\begin{array}{l}-N_{\mathrm{p}}=\frac{K_{\mathrm{p}}^{E}}{\mathrm{j} \omega}\left(\dot{U}_{\mathrm{p}_{\mathrm{L}}}-\dot{U}_{\mathrm{p}_{\mathrm{R}}}\right)+e_{\mathrm{p}} \hat{V}_{\mathrm{p}} \\ \hat{i}_{\mathrm{p}}=-e_{\mathrm{p}}\left(\dot{U}_{\mathrm{p}_{\mathrm{L}}}-\dot{U}_{\mathrm{p}_{\mathrm{R}}}\right)+j \omega \hat{C}_{\mathrm{p}}^{\varepsilon} \hat{V}_{\mathrm{p}}\end{array}\right.$,

where $K_{\mathrm{p}}^{E}=\bar{c}_{11}^{E} S_{\mathrm{p}} / l_{\mathrm{p}}, e_{\mathrm{p}}=-b \bar{e}_{31}$ and $\hat{C}_{\mathrm{p}}^{\varepsilon}=$ $\bar{\epsilon}_{33}^{\varepsilon} A_{\mathrm{p}} / h_{\mathrm{p}}$. It also reveals global properties, as $K_{\mathrm{p}}^{E}$ which is the stiffness of the patch at constant electric field, i.e. when the patch is short circuited $\left(\hat{V}_{\mathrm{p}}=0\right)$. Then, $e_{\mathrm{p}}$ represents the global coupling coefficient of the piezoelectric patch and $\hat{C}_{\mathrm{p}}^{\varepsilon}$ is its capacitance at constant strain. This constant strain condition is obtained when the difference between the end velocities is equal to zero $\left(\dot{U}_{\mathrm{p}_{\mathrm{L}}}-\dot{U}_{\mathrm{p}_{\mathrm{R}}}=0\right)$.

The global formulation given in (17) can be illustrated by the electric scheme introduced in figure 9 . The transformer, representing the piezoelectric coupling, is between the left and right branch, which are respectively related to the mechanical and electrical behavior. One can remark that this architecture corresponds to the one which couples two networks in figure 8 . As a consequence, it becomes possible to use discrete representations in order to study coupled problem involving piezoelectric elements.

\subsection{Electromechanical unit cell}

When analyzing an homogeneous rod fitted with a periodic distribution of piezoelectric patches, it is possible to extract the unit cell that repeats all along the structure. Here is considered the unit cell represented in figure 10, whose rod portion have a length $a$, a width $b$ and a thickness $h_{\mathrm{s}}$. It is symmetrically covered with a pair of piezoelectric patches of length $l_{\mathrm{p}}$, width $b$ and thickness $h_{\mathrm{p}}$, which are polarized in opposite directions. For the purpose of controlling longitudinal vibrations, the patches are electrically connected in parallel, as it was done by Thorp et al. [9]. Concerning the added electrical components, it is chosen to represent a general resonant configuration with two inductors $\hat{L} / 2$ on both sides of the pair of patches. Indeed, depending on the connection of the free ends of the inductors, it is possible to treat two different cases. First, a connection to the ground leads to the case of independent resonant shunts [5] involving inductors $\hat{L} / 4$. However, linking the inductors to those of the neighboring unit cells reveals an electric line where inductors $\hat{L}$ are placed between each pairs of patches. Both cases will be analyzed and related to the damping strategies presented in section 3 .

In order to study a finite structure consisting of $n$ unit cells as the one presented in figure 10, a first step is to define its transfer matrix. Contrary to previous studies on distributions of piezoelectric patches $[9,10,11,12,13,14,17,16,18,19,21,20]$, it is here chosen to firstly discretize the mechanical medium to simplify the problem and to suit with the discrete nature of the electrical network. The present goal is thus to transform the unit cell of the coupled problem into a discrete model having the same architecture than the analogues presented in figures 5 and 8 . Even if the

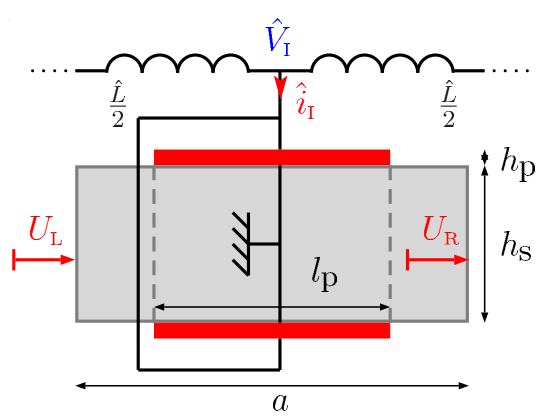

Figure 10. Unit cell of the electromechanical problem. 


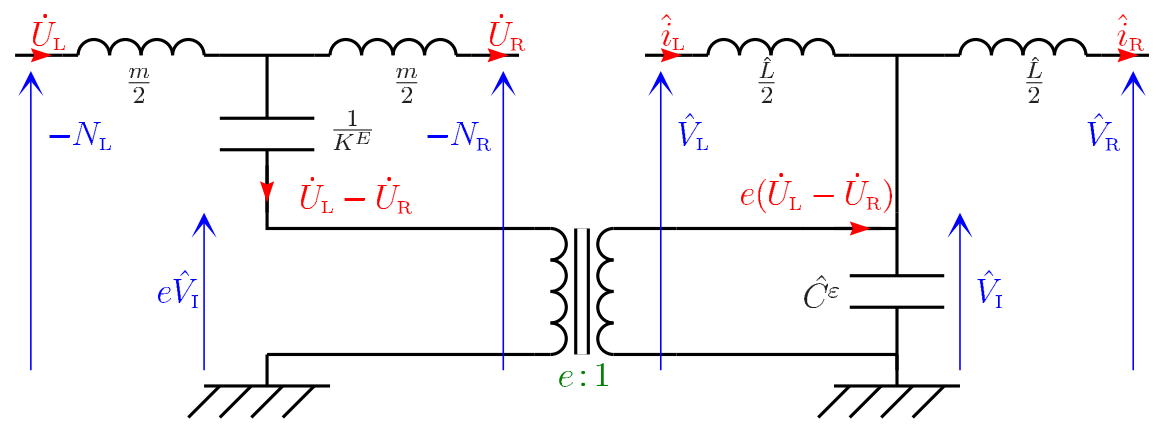

Figure 11. Discrete model of the electromechanical unit cell.

mechanical model is also suitable, it is chosen to focus on the electrical representation, which offers an easiest analysis. Consequently, the discrete model of the electromechanical unit cell is illustrated by the electric scheme of figure 11, where appear global constants that will need to be determined. The hatted notation is used in the right branch, which corresponds to the electrical structure added for vibration control. No hats are yet present in the left branch as it is related to the mechanical structure where vibrations need to be attenuated. From this model, it is already apparent that a multimodal control can be implemented by applying the modal coupling condition (14) adapted to the present configuration:

$\frac{1}{\hat{L} \hat{C}^{\varepsilon}}=\frac{K^{E}}{m}$.

It is also possible to consider independent resonant shunts by fixing $\hat{V}_{\mathrm{L}}$ and $\hat{V}_{\mathrm{R}}$ to zero. The target angular frequency will then be defined by the value of $2 / \sqrt{\hat{L} \hat{C}^{\varepsilon}}$. Consequently, the two resonance-based strategies defined in section 3 can be applied to the present coupled problem. It still remains to know the global properties of the discretized unit cell of figure 11.

\subsection{Global properties}

As seen in section 2, the masses $m$ involved in the mechanical lattice comes directly from the total mass of the continuous unit cell. If $\rho_{\mathrm{s}}$ and $\rho_{\mathrm{p}}$ are respectively the density of the rod structure and the density of the piezoelectric patches, it gives:

$m=\rho_{\mathrm{S}} S_{\mathrm{S}} a+2 \rho_{\mathrm{p}} S_{\mathrm{p}} l_{\mathrm{p}}$,

where $S_{\mathrm{S}}=h_{\mathrm{S}} b$. Then, it is observed from figure 11 that the stiffness $K^{E}$ of the lattice corresponds to the longitudinal stiffness of the unit cell when the pair of patches is short circuited $\left(\hat{V}_{\mathrm{I}}=0\right)$. In this situation, the equivalent Young modulus of the piezoelectric material is $Y_{\mathrm{p}}^{E}=\bar{c}_{11}^{E}$. The Young modulus of the $\operatorname{rod}$ being $Y_{\mathrm{s}}$, the stiffness $K^{E}$ is obtained from the continuous unit cell after considering that all the cross sections remain undeformed:

$$
\frac{1}{K^{E}}=\frac{l_{\mathrm{p}}}{Y_{\mathrm{s}} S_{\mathrm{s}}+2 Y_{\mathrm{p}}^{E} S_{\mathrm{p}}}+\frac{a-l_{\mathrm{p}}}{Y_{\mathrm{s}} S_{\mathrm{s}}} .
$$

For the electrical branch, the inductance $\hat{L} / 2$ corresponds directly to the value of the inductors connected to the patches but it remains to define the capacitance $\hat{C}^{\varepsilon}$ and the global coupling coefficient $e$. Concerning $\hat{C}^{\varepsilon}$, it is the capacitance of the pair of patches when $\dot{U}_{\mathrm{L}}-\dot{U}_{\mathrm{R}}$ is equal to zero. It is not simply equal to two times $\hat{C}_{\mathrm{p}}^{\varepsilon}$, the capacitance of a single isolated patch at constant stain, for two reasons. First, the two patches are no more free along the '2' direction because they are bonded to the rod. Secondly, even with $U_{\mathrm{L}}=U_{\mathrm{R}}=0$, the patches are not completely blocked along the ' 1 ' direction when $a \neq l_{\mathrm{p}}$. The analytic calculation of $\hat{C}^{\varepsilon}$ is thus non trivial and needs to take into account $3 \mathrm{D}$ effects as it was remarked by Maurini et al. [23]. The same problem appears when focusing on the global coupling coefficient $e$, which can not be obtained directly from a simple one dimensional model. However, this issue can be avoided by allowing a direct capacitance measurement.

The measure of $\hat{C}^{\varepsilon}$ is difficult in practice because it would require to fix the ends of one specific unit cell. Yet, the capacitance in free conditions $\hat{C}^{\sigma}$ is more easily reachable, as it can be obtained by direct measurement without any normal forces $\left(N_{\mathrm{L}}=N_{\mathrm{R}}=\right.$ $m=0)$. Moreover, figure 11 gives the following relation:

$\hat{C}^{\sigma}=\hat{C}^{\varepsilon}+\frac{e^{2}}{K^{E}}$

The global coupling coefficient $e$ is still unknown, which requires a new equation. To find this complementary information, it is first remarked that $Y_{\mathrm{p}}^{D}$, the equivalent piezoelectric Young modulus in open circuit, can be obtained. The condition of large wavelength compared to $l_{\mathrm{p}}$ is again considered in (16) and $D_{3}=0$ gives:

$Y_{\mathrm{p}}^{D}=\bar{c}_{11}^{E}+\frac{\bar{e}_{31}^{2}}{\bar{\epsilon}_{33}^{\varepsilon}}$. 
Then, the global stiffness in open circuit $K^{D}$ comes from (20) when replacing $Y_{\mathrm{p}}^{E}$ by $Y_{\mathrm{p}}^{D}$ and figure 11 links $K^{D}$ to the other global constants:

$K^{D}=K^{E}+\frac{e^{2}}{\hat{C}^{\varepsilon}}$.

At the end, the capacitance $\hat{C}^{\varepsilon}$ and the global coupling coefficient $e$ are obtained from (21) and (23):

$$
\left\{\begin{array}{l}
e=\sqrt{K^{E}\left(1-\frac{K^{E}}{K^{D}}\right) \hat{C}^{\sigma}} \\
\hat{C}^{\varepsilon}=\hat{C}^{\sigma} \frac{K^{E}}{K^{D}}
\end{array} .\right.
$$

Consequently, the discrete model of the unit cell is complete and its transfer matrix can be deduced from (13).

\subsection{Semi-continuous transfer matrix}

The transformation of the unit cell presented in figure 10 into its discrete model of figure 11 requires to approximate the continuous rod by a discrete lattice. As mentioned in section 2 , this is only valid when the considered wavelength is large compared to the length of the unit cells. However, it is possible to come back to a continuous mechanical medium by applying the reverse transformation described in (11). The unit cell can thus be described with a semi-continuous transfer matrix that respects both the continuity of the mechanical structure and the discrete nature of the electrical network:

$$
\left[\begin{array}{c}
\dot{U}_{\mathrm{R}} \\
\hat{i}_{\mathrm{R}} \\
N_{\mathrm{R}} \\
\hat{V}_{\mathrm{R}}
\end{array}\right]=\left[\begin{array}{cccc}
1-f & \frac{e}{K \hat{C}^{\varepsilon}} \hat{f} & \frac{\mathrm{j} \omega}{K} & \frac{\mathrm{j} e \omega}{K} \\
e f & 1-\Lambda \hat{f} & -\frac{j \mathrm{j} \omega}{K} & -j \Lambda \hat{C}^{\varepsilon} \omega \\
-\frac{2 K}{\mathrm{j} \omega} f\left(1-\frac{f}{2}\right) & -\frac{e}{\mathrm{j} \hat{C}^{\varepsilon} \omega} f \hat{f} & 1-f & -e f \\
\frac{e}{\hat{C}^{\varepsilon} \omega} f \hat{f} & \frac{2 \hat{f}}{\mathrm{j} \hat{C}^{\varepsilon} \omega}\left(1-\frac{\Lambda \hat{f}}{2}\right) & -\frac{e \hat{f}}{K \hat{C}^{\varepsilon}} & 1-\Lambda \hat{f}
\end{array}\right]\left[\begin{array}{c}
\dot{U}_{\mathrm{L}} \\
\hat{c}_{\mathrm{L}} \\
N_{\mathrm{L}} \\
\hat{V}_{\mathrm{L}}
\end{array}\right]
$$

where $f=1-\cos \left(\sqrt{m / K^{E}} \omega\right)$,

$K=K^{E}\left(\sqrt{m / K^{E}} \omega\right) / \sin \left(\sqrt{m / K^{E}} \omega\right), \hat{f}=\omega^{2} \hat{L} \hat{C}^{\varepsilon} / 2$ and $\Lambda=1+e^{2} /\left(K \hat{C}^{\varepsilon}\right)$. It can be remarked that the reverse transformation is applied on the homogenized mechanical constants $m$ and $K^{E}$. Consequently, the spatial discontinuity induced by the thickness of the piezoelectric patches doesn't appear in the formulation. This model is thus not suitable to track the band gap phenomenon $[9,12,13]$ but it better fits the continuous behavior than the lattice model.

The transfer matrix presented in (25) gives a relation between the electromechanical state vectors containing the velocities, intensities, normal forces and voltages at both ends of the unit cell. After defining mechanical and electrical boundary conditions, it becomes possible from (3) to study a finite structure consisting of $n$ coupled unit cells. The multimodal control strategy can be implemented by applying (18) together with analogue boundary coditions at the end of the two structures. Therefore, the network being tuned to fit the modes of the equivalent mechanical lattice, it will obviously not match exactly the modes of the continuous rod. However, as presented in section 2, 10 unit cells per wavelength gives a frequency difference of less than $2 \%$. So, this mistuning is negligible for a sufficiently high number of unit cells. Finally, the independent resonant shunts configuration can also be analyzed from the previous general formulation. The $4 \times 4$ transfer matrix presented in $(25)$ is condensed in a $2 \times 2$ matrix by setting $\hat{V}_{\mathrm{L}}$ and $\hat{V}_{\mathrm{R}}$ equal to 0 . At the end, (3) is still employed to obtain the global behavior the finite electromechanical structure.

\section{Experiments and discussion}

The two control strategies based on independent resonant shunts and on a multi-resonant network are applied on a rod which is periodically equipped with piezoelectric patches. The experimental results are compared to the numerical results obtained with the semi-continuous transfer matrix. Both strategies are evaluated by taking into account their practical implementation.

\subsection{Experimental setup}

The main structure is a one meter duralumin rod, which is periodically covered with $n=20$ pairs of piezoelectric patches. Referring to figure 10, the geometry of the setup is presented in table 1 . Concerning the piezoelectric material, the PIC 151 ceramic is selected in order to keep the possibility to extend some of the results presented in previous studies [8, 22]. The properties of the PIC 151 are also described in table 1, which reports values appearing in the PI Ceramic data sheet. The resulting structure is suspended by elastic straps in order to tend to a freefree configuration. Then, as presented in figure 12, a suspended shaker is connected to one end of the rod through an impedance head that measures the acceleration and the transmitted force. At the other end, a scanning laser vibrometer measures the velocity. A white noise excitation is generated and two signals are analyzed: the velocity acquired by the vibrometer and the force measured by the impedance head. A last step consists in obtaining the transfer function between the velocity and the force signals. To this end, a fast Fourier transform is performed on a $11.5 \mathrm{kHz}$ frequency range with a $2 \mathrm{~Hz}$ resolution.

The analysis is firstly performed without adding any electrical components on the piezoelectric patches. The experimental frequency response functions are compared to the results obtained with the purely mechanical homogenized model computed from (2) and (6). It is seen that the model can be improved by 


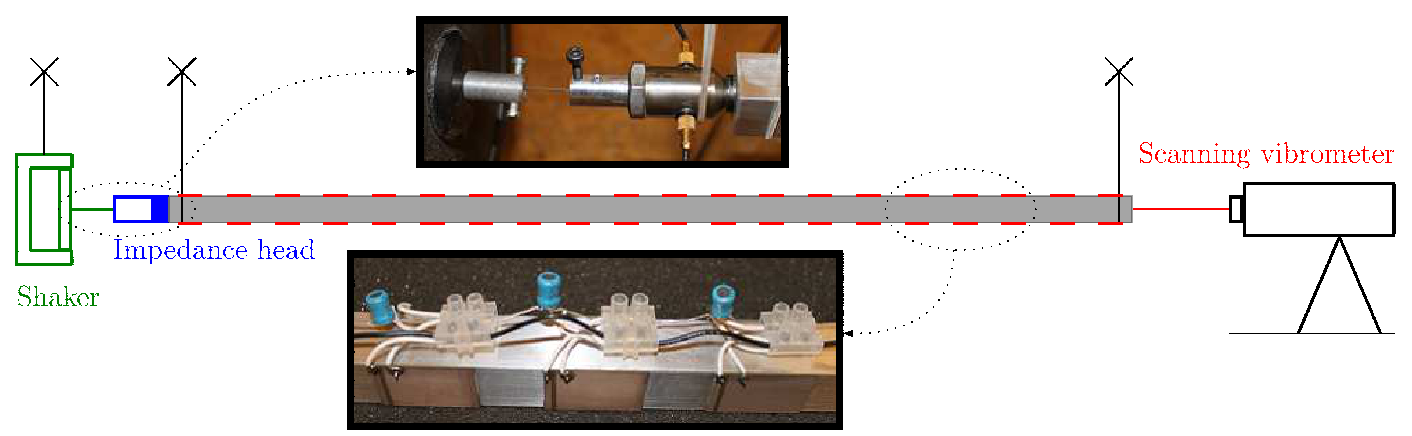

Figure 12. Experimental setup.

Table 1. Geometry and material properties.

\begin{tabular}{lll}
\hline & Rod (AU4G) & Patches (PIC 151) \\
\hline Length & $l_{\mathrm{s}}=N a=20 \times 5 \mathrm{~cm}$ & $l_{\mathrm{p}}=3 \mathrm{~cm}$ \\
Width & $b=2 \mathrm{~cm}$ & $b=2 \mathrm{~cm}$ \\
Thickness & $h_{\mathrm{S}}=2 \mathrm{~cm}$ & $h_{\mathrm{p}}=0.5 \mathrm{~mm}$ \\
\hline Density & $\rho_{\mathrm{S}}=2780 \mathrm{~kg} / \mathrm{m}^{3}$ & $\rho_{\mathrm{p}}=7800 \mathrm{~kg} / \mathrm{m}^{3}$ \\
Young modulus & $Y_{\mathrm{S}}=73.9 \mathrm{GPa}$ & $1 / s_{11}^{E}=66.7 \mathrm{GPa}$ \\
Charge constant & - & $d_{31}=-210 \mathrm{pC} / \mathrm{N}$ \\
Permittivity & - & $\epsilon_{33}^{\sigma}=21.2 \mathrm{nF} / \mathrm{m}$ \\
\hline
\end{tabular}

considering three new elements related to the setup. First, it is remarked that the impedance head is not directly connected to the rod as a small connecting element is used for practical purpose. This induces that the measured force $N_{\text {mes }}$ is not exactly equal to the normal force $N_{0}$ really applied to the rod. Yet, those two forces can be related by remarking that the displacement of the connecting element is equal to the displacement of the excited end of the rod $q_{0}$. This gives:

$\frac{N_{\text {mes }}}{N_{0}}=1+\omega^{2} m_{\text {add }} \frac{q_{0}}{N_{0}}$,

where $m_{\text {add }}$ is the mass of the connecting element which was measured equal to 4 grams. This force ratio is thus used to correct the frequency response function obtained from (3). Secondly, it is observed that the stiffness added by the patches is slightly lower than the theoretical value obtained with the longitudinal model. This is due to $3 \mathrm{D}$ effects and to the unconsidered flexibility induced by the glue that bond the patches to the rod. The loss of stiffness is corrected in the model by adding a coefficient $\gamma$ that modify the value of the Young modulus in short and open circuit $\left(\gamma Y_{\mathrm{p}}^{E}\right.$ and $\left.\gamma Y_{\mathrm{p}}^{D}\right)$. The coefficient $\gamma$ is fixed to 0.86 to approach the experimental results. At the end, the third add in the model concerns the damping. It is observed experimentally that the maximum velocity at resonance decrease with an increase of the mode number. This can be modeled by using equivalent complex Young moduli on the form $Y^{\star}=Y(1+j \eta(\omega))$, where $\eta(\omega)=\alpha \omega$. The coefficient $\alpha$ is evaluated to $3 \cdot 10^{-8}$ in order to get close to the four first maxima of the velocity with open circuited piezoelectric patches.

Concerning the electrical components, as it was chosen to focus on a completely passive control solution, they are only selected in standard series with a $10 \%$ tolerance. It was observed that the internal resistance of the standard inductors is non negligible and often too high for the present application. Consequently, the inductors were chosen as low resistive as possible, which justify the absence of damping optimization. In the same time, no specific resistors need to be added, which simplifies the network. The suppliers of standard electrical components proposing a set of discrete values, it is not always possible to find inductors that suits with the inductance $\hat{L}$ required for a modal coupling. However, it is possible to finalize the tuning by adding capacitors in parallel to the piezoelectric patches. This increases the capacitance $\hat{C}^{\varepsilon}$ and gives a new degree of freedom in the choice of $\hat{L}$. Nano or tenth of nano Farad are available in metallized polyester film capacitors series. This suits with the value of $\hat{C}^{\varepsilon}$ which was evaluated to $36 \mathrm{nF}$ without added capacitors.

\subsection{Independent resonant shunts}

The first experiment consists in the implementation of the first control strategy, which involves independent resonant systems. The periodicity of the structure is kept by distributing identical resonant shunts all along the rod. The first resonance of the free-free rod is chosen as the target of the control strategy (2500 $\mathrm{Hz}$ ). Consequently, the electrical resonance needs to be tuned to this specific frequency. Direct measurements on a set of 20 inductors selected in a $100 \mathrm{mH}$ series give an average inductance $\hat{L} / 4=96 \mathrm{mH}$ and an average serial resistance $\hat{R}_{\mathrm{S}}=80 \Omega$. For this inductance, a tuning at $2500 \mathrm{~Hz}$ requires to increase $\hat{C}^{\varepsilon}$ by $6.2 \mathrm{nF}$. This is done by placing individual capacitors in parallel to the pairs of piezoelectric patches. The frequency response function corresponding to the end velocity 


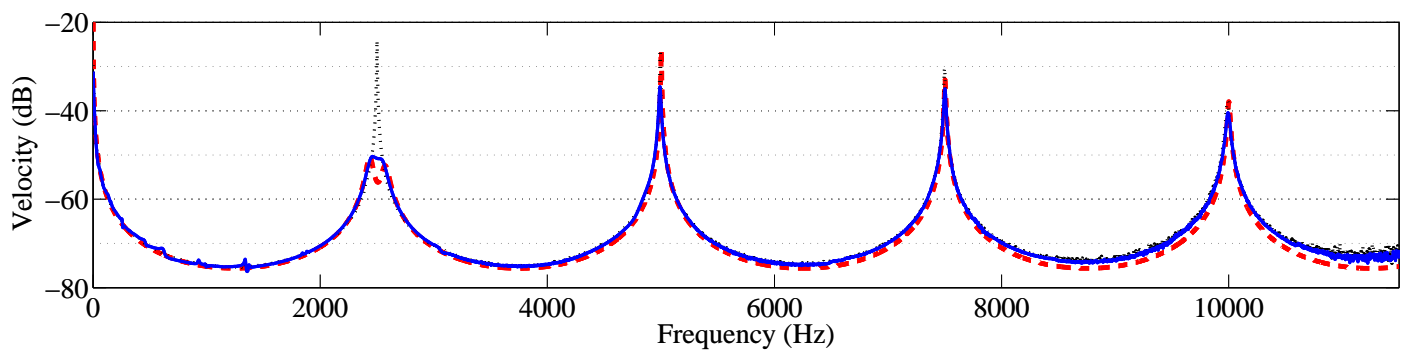

Figure 13. Frequency responses for the distributed shunts strategy - ( . ) for the experiment with open circuits, (-) for the experiment with resonant shunts, $(--)$ for the transfer matrix model with resonant shunts.

obtained with a unit exiting force is presented in figure 13. The four first modes of the free-free rod are observed in the $11.5 \mathrm{kHz}$ frequency range. One can see that the passive damping system gives a reduction of about $25 \mathrm{~dB}$ on the velocity amplitude of the $1^{\text {st }}$ resonance. On the contrary, the other resonances only present a limited attenuation. This agrees with the frequency response obtained in figure 4 for a similar strategy with lattice models.

The response of the longitudinal model involving the $2 \times 2$ matrix condensed from (25) is compared to the experimental results. As performed in section 2 for the tuned masses, the damping is taken into account by replacing the tuned inductance by $\hat{L}^{\star} / 4=\hat{L} / 4-$ $j \hat{R}_{\mathrm{S}} / \omega$. This corresponds to the equivalent inductance model that is obtained when adding a resistor in series with an inductor. The expectations concerning the velocity reduction on the fist mode are fully satisfied as there is a difference of less than $1 \mathrm{~dB}$ between the two maxima. Nevertheless, the theoretical curve presents a local minimum, which is a characteristic of a slightly underdamped tuned mass system $[5,6,8]$. This double peak shape doesn't clearly appear in the present experiment. It is observed a more damped behavior than the one predicted by the model. Actually, the $10 \%$ tolerance of the standard passive components creates small tuning differences all along the twenty shunts. Thus, as introduced in [9], the electrical resonances are distributed around an average frequency value, which flattens the experimental frequency response function. Anyway, a significant vibration reduction is obtained on one particular mode with a completely passive system. Yet, it remains the question of the frequency range of interest. For a constant capacitance, the resonance frequency of the shunt is proportional to $1 / \sqrt{\hat{L}}$. Consequently, if the target frequency is divided by two, the inductance needs to be four times higher. This becomes a real problem when looking at low frequencies. Indeed, it would requires to use large and heavy components that are no more in the scope the standard passive series considered in this study. Increasing the number of piezoelectric patches doesn't solve the problem as it can be seen from (17) that the capacitance is roughly proportional to $1 / n$. As a consequence, the strategy involving a succession of several similar resonant shunts have significant limits on its frequency range of application.

\subsection{Multi-resonant network}

The second experiment is related to the validation of the multimodal damping strategy. For the geometry and material properties described in table 1, the application of the modal coupling condition (18) gives an inductance $\hat{L}$ around $2.8 \mathrm{mH}$. As it was possible to find a set of components presenting this average inductance, no capacitors need to be added. All the capacitive behavior is supported by the pairs of piezoelectric patches. So, the electrical network was realized by simply linking successive unit cells with the selected inductors. The two ends of the line are then closed by a $\hat{L} / 2$ inductance. This allows to respect the unit cell architecture and the zero voltage boundary conditions which are the equivalent of the free-free mechanical boundary conditions of the rod.

It is possible to control the tuning of the electrical network before looking at its effect on vibration reduction. A white noise voltage is applied at one end of the network and voltage is measured in the middle of the network. This gives the electrical frequency response function presented in figure 14 . The mechanical analogue of this experiment would be to measure the force in the middle of the rod when applying a force at one end. As a free-free configuration is analyzed, the middle of the rod is a node for the even force modes. The same remark applies to the voltage as the electrical network was designed to tend to the analogue of the free-free rod. This is confirmed by the electrical frequency response function where only the odd resonances appears. A second remark concerns the sharp antiresonances that can be observed on the response. They corresponds to an energy transfer from the network to the rod at the mechanical resonances. Consequently, the tuning of the network is electrically validated by controlling that the mechanical resonances 


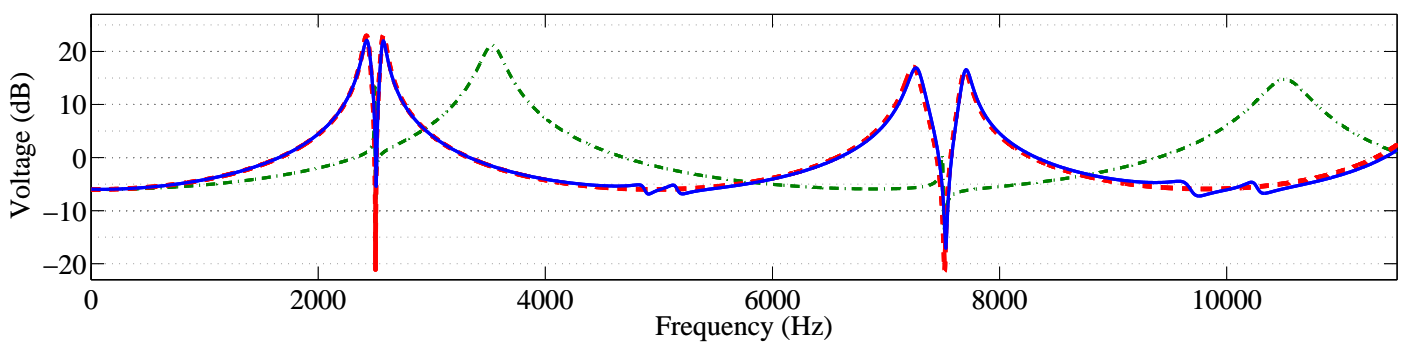

Figure 14. Electrical frequency responses for the multimodal damping strategy - (-) for the experiment with an optimal network, $(--)$ for the transfer matrix model with an optimal network, $\left(--_{-}\right)$for the transfer matrix model with a non-optimal network.

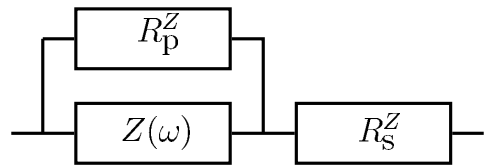

Figure 15. Model for the electrical components.

are centered on the electrical resonances.

The $4 \times 4$ matrix in $(25)$ can be used to compare the semi-continuous transfer matrix model to the experimental results on the voltage response. It was found that an accurate numerical result is obtained when using both a serial and a parallel resistance, $R_{\mathrm{S}}^{Z}$ and $R_{\mathrm{p}}^{Z}$, when modeling the electrical components. This model, presented in figure 15, applies on the inductors with $Z(\omega)=j \hat{L} \omega$ and on the capacitors with $Z(\omega)=1 /\left(j \hat{C}^{\varepsilon} \omega\right)$. From this representation, it is obtained the equivalent electrical formulations that are introduced in the transfer matrix in order to consider damping:

$$
\left\{\begin{array}{l}
\hat{L}^{\star}=\frac{R_{\mathrm{p}}^{L} \hat{L}}{R_{\mathrm{p}}^{L}+j \hat{L} \omega}-\frac{R_{\mathrm{S}}^{L}}{\omega} \\
\frac{1}{\hat{C}^{\star}}=\frac{\mathrm{j} R_{\mathrm{p}}^{C} \omega}{1+j R_{\mathrm{p}}^{C} \hat{C}^{\varepsilon} \omega}+j R_{\mathrm{S}}^{C} \omega
\end{array} .\right.
$$

The following average values were measured directly on the electrical components in the considered frequency range: $R_{\mathrm{S}}^{L}=1.3 \Omega, R_{\mathrm{p}}^{L}=12 k \Omega, R_{\mathrm{S}}^{C}=2.8 \Omega$ and $R_{\mathrm{p}}^{C}=180 k \Omega$. Those values are then used to compute the electrical frequency response function. It can be remarked in figure 14 that the theoretical antiresonances are deeper than the experimental ones. In fact, for practical reasons, the rod was placed on a foam carpet during electrical measurements, which added mechanical damping. Apart from antiresonances, the numerical and experimental curves are sufficiently close to validate the transfer matrix model, that can then be used for a network evaluation purpose. Indeed, it is also represented in figure 14 a numerical frequency response function for a network that doesn't respect the coupling condition (18) (half of the required inductance). In this last case, it is clearly observable that the electrical resonances are not centered on the mechanical ones.

Once the electrical network is correctly tuned, the effect of the multimodal coupling on the mechanical vibrations can be observed. Figure 16 represents the experimental frequency response functions with a tuned network and without any added electrical components. The performances are significant as the reduction is close to $25 \mathrm{~dB}$ for the first three resonances. This clearly highlights the power of a multimodal damping by the use of a passive electrical network. It can still be remarked that a slight increase of the damping would flattens the response and add a complementary reduction of few decibels. The lowest resistive $2.8 \mathrm{mH}$ inductors found in standard series have thus a serial resistance which is finally a bit too low for the present application. In any case, the experimental results are again compared to the matrix computation. The two response are again very similar, which gives a strong support for the semicontinuous transfer matrix formulation for vibration control analysis. It can be noticed that the only adjusted model parameters are $\gamma$ and $\alpha$, the stiffness loss and the viscous damping coefficients, which are determined from the purely mechanical responses. All the other constants come from direct measurements or from data sheets. Moreover, the vibration reduction effect of the present control system is so important that the adds of $\gamma$ and $\alpha$ in the model doesn't induce noticeable effect when the modal coupling is functional.

Concerning the tuning of the electrical network, it can be seen from figure 16 that the induced antiresonances are placed at lower frequencies than the initial mechanical resonances. This effect is not clearly observable for the first mode but is more pronounced for higher mode numbers. It significantly exceeds the resonance shift due to the addition of damping. This increasing mistuning is due to the fact that the electrical network is a discrete structure. As seen in section 2, for a same wavelength equal to 10 unit cells, there is a frequency difference close to $2 \%$ between the continuous rod and its discrete analogue. With 20 


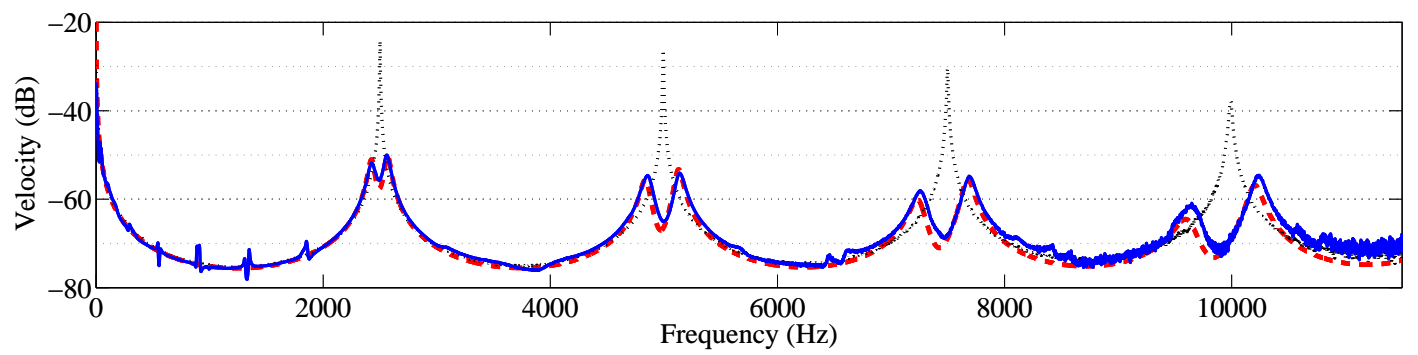

Figure 16. Mechanical frequency responses for the multimodal damping strategy - $(\cdots)$ for the experiment without network, $(-)$ for the experiment with an optimal network and (--) for the transfer matrix model with an optimal network.

cells on a free-free structure, this frequency difference is obtained on the fourth modes. The mistuning remains limited but becomes clearly non-negligible for higher mode. If a larger number of modes needs to be controlled, it is still possible to increase the number of unit cells. This simply enhances the frequency range in which the discretized behavior remains close the continuous one.

At last, it is seen from the modal coupling condition (18) and the definition of the global constants that the required inductance is approximately proportional to the inverse of the number of unit cells: $\hat{L} \propto 1 / n$. This conclusion was already highlighted by Maurini et al. [18] from the analysis an electrical continuum. The relation is here illustrated for the discrete formulation but still induces that the increase of the number of unit cells leads to lower values of the required inductance. As a consequence, it becomes possible to look at lower frequency applications without being limited by the values proposed in standard passive series of inductors. This strongly increases the potential of the multimodal damping strategy, which was already attractive for its broadband capabilities.

\section{Conclusions}

It is observed that a continuous rod can be seen as a periodic structure and analyzed through a transfer matrix formulation. This formulation is also relevant when modeling a rod with a lattice of point masses that are serially connected with springs. The differences between the continuous and the discrete models is presented. The lattice period is compared to the considered wavelength as the unit cells need to be sufficiently small to approximate a continuous behavior. Secondly, it is looked for solutions that could lead to the conversion of vibration energy from a main lattice to a control system. A first strategy consists in using independent tuned mass dampers that are distributed all along the structure. A constant tuning gives the possibility to target one specific frequency, which corresponds to the resonance of the added systems. Then, a multimodal control strategy is introduced. This can be obtained by connecting a main structure to its modal equivalent. The unit cell that couple two lattices is presented and simple modal coupling conditions are obtained. With similar dispersion relations and boundary conditions, it is possible to reach a multimodal tuned mass damping that apply on all the natural frequencies of the main structure. The two resonance-based strategies are transposed in the electrical domain by using a direct electromechanical analogy. The corresponding unit cell are defined as well as the way to obtain the electrical transfer matrices.

In a context of rod vibration control with piezoelectric patches, the electromechanical analogy implies that the independent tuned mass dampers strategy is the analogue to the use of distributed resonant shunts. Moreover, the electrical analogue of a mechanical lattice points out the fact that a multimodal strategy can be achieved by connecting an electrical network to the continuous rod. After defining a global model for the piezoelectric elements, a unit cell of the electromechanical problem is presented. The global properties of this coupled unit cell are then defined from the equivalent discrete electrical architecture. All of this gives the possibility to define a $4 \times 4$ transfer matrix that suits with the analysis of longitudinal wave propagation for the two control strategies. A novel semi-continuous transfer matrix formulation is presented in order to consider both the continuity of the rod and the discrete nature of the electrical network. Experiments on a rod equipped with 20 pairs of piezoelectric patches validate this model for both mechanical and electrical responses. Furthermore, the two control strategies are implemented with standard passive components. The multimodal damping strategy is clearly validated as the performances are considerable on all the observed modes. Another strong interest of this strategy is the fact the inductance can be kept sufficiently low by increasing the number of unit cells. This differs from the more classical independent shunts strategy that becomes impractical when looking at low frequencies.

Finally, it is shown that the use of a network link- 
ing several piezoelectric elements can offer substantial benefits, for both control performances and practical implementation. Periodic longitudinal configurations can be modeled with a transfer matrix formulation involving electromechanical state vectors. All of this is here applied to the control of longitudinal waves and it will then be extended to transverse propagation. This future work should lead to an experimental validation of a multimodal control involving a bending beam coupled to a dispersive electrical network.

\section{Acknowledgments}

This work was funded by the French Ministry of National Education, Higher Education and Research through a three year scholarship for doctoral studies related to structural damping with piezoelectric devices.

[1] Mead D M 1996 J. Sound Vib. 190 495-524

[2] Brillouin L 1946 Wave propagation in periodic structures (New-York: McGraw-Hill)

[3] Bloch A 1945 J Inst. Electr. Eng. 92 157-69

[4] Beranek L L 1954 Acoustics (New-York: McGraw-Hill)

[5] Hagood N W and von Flotow A 1991 J. Sound Vib. 146 243-68.

[6] Den Hartog J P 1940 Mechanical vibrations (New-York: McGraw-Hill)

[7] Liu K and Liu J 2005 J. Sound Vib. 284 1181-89.

[8] Thomas O, Ducarne J and Deü J-F 2012 Smart Mater. Struct. 21015008.

[9] Thorp O, Ruzzene M and Baz A 2001 Smart Mater. Struct. 10979.

[10] Airoldi L and Ruzzene M 2011 New J. Phys. 13113010.

[11] Airoldi L and Ruzzene M 2011 J. Int. Mater. Sys. Struct. 22 1567-79

[12] Wang G, Chen S and Wen J 2011 Smart Mater. Struct. 20 015026.

[13] Wang G, Wang J, Chen S and Wen J 2011 Smart Mater. Struct. 20125019.

[14] Beck B S, Cunefare K A, Ruzzene M and Collet M $2011 \mathrm{~J}$. Int. Mater. Sys. Struct. 22 1177-87.

[15] Yu H, Wang K W and Zhang J 2006 J. Sound Vib. 295 $246-65$.

[16] dell'Isola F, Maurini C and Porfiri M 2004 Smart Mater. Struct. 13 299-308.

[17] Vidoli S and dell'Isola F 2000 Acta Mech. 141 37-50.

[18] Maurini C, dell'Isola F and Del Vescovo D 2004 Mech. Syst. Signal Process. 18 1243-71.

[19] Andreaus U, dell'Isola F and Porfiri M 2004 J. Vib. Contr. $10625-59$.

[20] Lu Y and Tang J 2012 J. Sound Vib. 331 3371-85.

[21] Bisegna P, Caroso G and Maceri F 2006 J. Sound Vib. 289 908-37.

[22] Thomas O, Deü J-F and Ducarne J 2009 Int. J. Numer. Meth. Eng. 80 235-68.

[23] Maurini C, Pouget J and dell'Isola F 2006 Comput. Struct. 84 1438-58. 